\title{
Thermographic Stall Detection Using Model-Based Evaluations of the Surface Temperature Response to Oscillating Fluid Temperatures
}

\author{
Felix Oehme ${ }^{1}$, Michael Sorg ${ }^{1}$, Andreas Fischer ${ }^{1}$ \\ ${ }^{1}$ University of Bremen, Bremen Institute for Metrology, Automation and Quality Science \\ Linzer Str. 13, 28359, Bremen, Germany \\ Correspondence: f.oehme@bimaq.de
}

\begin{abstract}
Summary:
In the operation of wind turbines, flow separation on rotor blades causes performance losses, structural loads and acoustic emissions. To unambiguously detect flow separation using thermographic images despite a low thermal contrast, a model-based signal processing approach is presented. The proposed signal processing approach evaluates the amplitudes of the surface temperature response to oscillating input signals selectively. Due to the direct correlation between the amplitude information and the flowdependent heat transfer coefficient, an unambiguous detectability of stall is achieved.
\end{abstract}

Keywords: IR thermography, thermographic boundary layer measurements, flow separation, turbulent flow separation, flow separation on rotor blades

\begin{abstract}
Introduction
Flow separation on rotor blades of wind turbines with pitch control is an undesirable flow state. It causes a decrease of torque producing lift, an increase in drag and acoustic emissions as well as structural loads. A measurement method with the potential to investigate the origin of flow separation on real wind turbines in operation is thermographic flow visualization using infrared light [1]. However, due to the low thermal contrast between turbulent and separated flow regions, the thermographic visualization of flow separation requires measurement conditions with a high temperature difference between the rotor blade surface and the incoming flow. For free field measurements, the available thermal contrast is only based on the absorbed solar radiation. Therefore, a signal processing approach is required which provides a high identifiability for flow separation.
\end{abstract}

The state-of-the-art signal processing approach for measurements with low thermal contrast evaluates the dynamic change of the surface temperature in thermographic image series [2]. However, the signal measurement chain is not considered, and a distinct evaluation of the influences on the dynamic surface temperature is missing. Thus, designing a model-based signal processing approach which provides a high interpretability for the unambiguous stall detection is an open task.
Therefore, the rotor blade surface response with respect to boundary layer flow characteristics and temporal fluctuations of the heat inputs is modeled using system theory. Based on the system modelling, a harmonic analysis of temporal surface temperature fluctuations around predefined operating points is then derived.

\section{Measurement Principle}

The proposed harmonic signal processing approach considers the small-scale oscillations of the surface temperature around an operating point as a thermodynamic response to the oscillating temperature of the incoming flow. The system's input-output relation shows the behavior of a linear time-invariant system and can be evaluated with an analysis of the amplitude attenuation of the output signal for selected frequency components. Here, the amplitude attenuation is a function of the selected frequency, thermal material parameters and the flow-dependent heat transfer coefficient. The resulting analogy between the spatial amplitude distribution and the distribution of the flow-dependent heat transfer coefficient enables a subsequent identification of the different flow regions including stall.

In order to calculate the amplitude of the output signal, a pixelwise discrete Fourier transformation is conducted for thermographic image series. Compared to the classical approach for thermographic flow visualization of evaluating temperature fields, the harmonic signal 
processing approach provides a significant reduced influence regarding undesired cross-sensitivities. While the dynamic behavior of the heat inputs is considered as desirable input signal, the undesired influence of heat conduction and the initial rotor blade temperature distribution is reduced by the dynamic behavior of the output signal. As a result, the amplitude information correlates directly with the flow-dependent heat transfer coefficient.

\section{Results}

In order to prove the potential of the harmonic signal processing approach, wind tunnel measurements with a wind turbine rotor blade profile and free field similar Reynolds numbers are performed. The location of the different flow states is validated by surface pressure measurements. Fig. 1 shows the processed thermographic image series for the harmonic as well as the mean temperature signal processing approach as reference. Both approaches enable a distinction between the laminar/turbulent and the turbulent/separated flow regions. Compared to the mean temperature approach, the harmonic approach suffers from an increasing variance of the evaluated amplitudes within each flow region as a result of analyzing the small-signal behavior of the surface temperature oscillation as well as the reduced influence of heat conduction. In consequence, the contrast between the different flow regions is reduced, while flow-dependent features are visible.

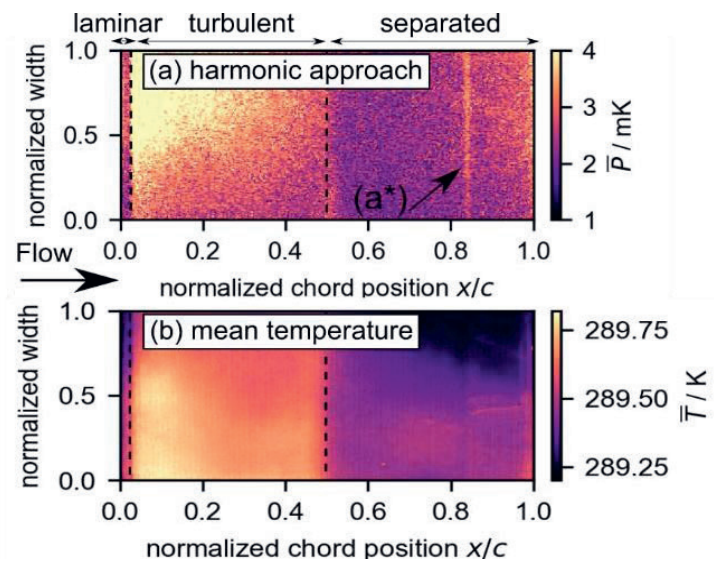

Fig. 1 Processed thermographic image series by the harmonic (a) and the mean temperature signal processing approach (b). (a*): Surface modification on the measuring object.

In order to prove the desired identifiability of flow regions based on flow-dependent features, Fig. 2 shows the expected course of the heat transfer coefficient based on theory (dotted) and experiments (dashed) over the normalized chord position as well as the normal to the flow direction averaged profiles of the presented signal processing approaches. Considering the results of the mean temperature approach, the mean temperature over the normalized chord position shows the expected signal behavior only for the transition regions. Within each flow region, the mean temperature course is mainly influenced by lateral heat conduction, characteristic separation features are not detectable.

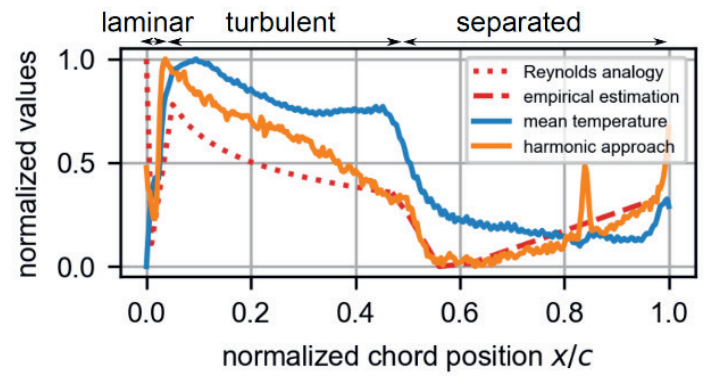

Fig. 2 Comparison of the expected (dotted, dashed) course of the heat transfer coefficient over the chord position and the measured results (solid line) of the mean temperature and the harmonic signal processing approach.

In contrast, the amplitude course of the harmonic approach reflects the theoretical estimations of the Reynolds analogy for the laminar and the turbulent flow region [3]. Inside the laminar and the turbulent flow region, a decreasing amplitude occurs as a result of an increasing boundary layer thickness. Flow separation is unambiguously identifiable based on a global amplitude minimum, a plateau near the separation point and an increasing amplitude inside the separated region. The increasing amplitude and the heat transfer coefficient over the cord position is induced by grown vortices and thus a typical feature for separated flow regions [4].

In conclusion, the proposed harmonic signal processing approach leads to an enhanced identifiability of flow separation.

The authors gratefully acknowledge the financial support of the Deutsche Forschungsgemeinschaft (DFG project number FI1989/5-1)

\section{References}

[1] E. Gartenberg, A. S. Roberts Jr., Twenty-five years of aerodynamic research with infrared imaging, Journal of Aircraft 2, 161-171 (1992), doi: $10.2514 / 3.46140$

[2] C. Dollinger, N. Balaresque, M. Sorg; A. Fischer, IR thermographic visualization of flow separation in applications with low thermal contrast, Infrared Physics \& Technology, 254-264 (2018), doi: 10.1016/j.infrared.2017.12.001

[3] H. Schlichting, K. Gersten, Boundary-Layer theory, Springer Berlin Heidelberg 9. edition (2017), doi: 10.1007/978-3-662-52919-5

[4] E. Gartenberg, A. S. Roberts Jr., Airfoil transition and separation studies using an infrared imaging system, Journal of Aircraft 4, 225-230 (1991), doi: $10.2514 / 3.46016$ 